\title{
Cenários Climáticos e Produtividade do Algodão no Nordeste do Brasil. Parte I: Calibração e Validação do Modelo Agrometeorológico
}

\author{
Fabrício Daniel dos Santos Silva ${ }^{1}$ (D), Rafaela Lisboa Costa $^{1}$ (D), \\ Rodrigo Lins da Rocha Júnior ${ }^{1}$ (D), Heliofábio Barros Gomes ${ }^{1}$ (D), Pedro Vieira de Azevedo ${ }^{2}$ (D), \\ Vicente de Paulo Rodrigues da Silva ${ }^{2}$ (D), Leonardo Amaral Monteiro ${ }^{3,4}$ \\ ${ }^{1}$ Instituto de Ciências Atmosféricas, Universidade Federal de Alagoas, Maceió, AL, Brasil. \\ ${ }^{2}$ Unidade Acadêmica de Ciências Atmosféricas, Universidade Federal de Campina Grande, \\ Campina Grande, PB, Brasil. \\ ${ }^{3}$ Departamento de Engenharia Agrícola, Universidade de Campinas, Campinas, SP, Brasil. \\ ${ }^{4}$ Department of Crop Sciences, Swedish University of Agricultural Sciences, Umeä, Sweden.
}

Recebido em: 6 de Julho de 2020 - Aceito em: 21 de Outubro de 2020

\begin{abstract}
Resumo
Esta pesquisa teve como objetivo calibrar e validar um modelo agrometeorológico de penalização por déficit hídrico aplicado à cultura do algodão herbáceo em 23 municípios da região Nordeste. Os resultados mostraram diferentes cenários, com produtividades variando de extremamente baixas, com média anual inferior a $500 \mathrm{~kg} / \mathrm{ha}$, a extremamente altas, com média anual superior a $3500 \mathrm{~kg} / \mathrm{ha}$. O processo de calibração do modelo agrometeorológico levou em consideração o nível de produtividade máxima tecnológica, estimada a partir de um ajuste estatístico baseado na série de dados das produtividades reais observadas, permitindo simulações satisfatórias dos valores disponibilizados pelo IBGE. Nos municípios com produtividades muito baixas até $500 \mathrm{~kg} / \mathrm{ha}$, a produtividade média observada (PMO) e simulada (PSO) foi de 333 e $361 \mathrm{~kg} / \mathrm{ha}$ com erro médio absoluto (EMA) de $\pm 56 \mathrm{~kg} / \mathrm{ha}$, para municípios com produtividades baixas entre 500 e $1000 \mathrm{~kg} / \mathrm{ha}$, a PMO e PSO foi de 774 e $814 \mathrm{~kg} / \mathrm{ha}$ com EMA de $\pm 172 \mathrm{~kg} / \mathrm{ha}$, nos municípios com produtividades medianas entre 1000 e $2000 \mathrm{~kg} / \mathrm{ha}$ foi de 1358 e $1400 \mathrm{~kg} / \mathrm{ha}$ com EMA de $\pm 198 \mathrm{~kg} / \mathrm{ha}$, e nos municípios com altas produtividades, acima de $2000 \mathrm{~kg} / \mathrm{ha}$, a PMO e PSO foi de 3233 e $3357 \mathrm{~kg} / \mathrm{ha}$ com EMA de $\pm 319 \mathrm{~kg} / \mathrm{ha}$, respectivamente.
\end{abstract}

Palavras-chave: algodão herbáceo, déficit hídrico, semiárido, produtividade.

\section{Climate Scenarios and Cotton Yield in Northeast Brazil. Part I: Calibration and Validation of the Agrometeorological Model}

\begin{abstract}
This research aimed to calibrate and validate an agrometeorological model of penalization for water deficit applied to the herbaceous cotton culture in 23 municipalities in the Northeast region. The results showed different scenarios, with yield varying from extremely low, with an annual average of less than $500 \mathrm{~kg} / \mathrm{ha}$, to extremely high, with an annual average of more than $3500 \mathrm{~kg} / \mathrm{ha}$. The calibration process of the agrometeorological model took into account the level of maximum technological productivity, estimated from a statistical adjustment based on the data series of the actual observed yield, allowing satisfactory simulations of the values made available by IBGE. In municipalities with very low yield up to $500 \mathrm{~kg} / \mathrm{ha}$, the average observed (AOY) and simulated (ASY) yield was 333 and $361 \mathrm{~kg} / \mathrm{ha}$ with mean absolute error (MAE) of $\pm 56 \mathrm{~kg} / \mathrm{ha}$, for municipalities with yield between 500 and $1000 \mathrm{~kg} / \mathrm{ha}$, the AOY and ASY was 774 and $814 \mathrm{~kg} / \mathrm{ha}$ with MEA of $\pm 172 \mathrm{~kg} / \mathrm{ha}$, in the municipalities with median yield between 1000 and $2000 \mathrm{~kg} / \mathrm{ha}$, the AOY and ASY was 1358 and $1400 \mathrm{~kg} / \mathrm{ha}$ with MAE of $\pm 198 \mathrm{~kg} / \mathrm{ha}$, and in municipalities with high yield, above $2000 \mathrm{~kg} / \mathrm{ha}$, the AOY and ASY was 3233 and $3357 \mathrm{~kg} / \mathrm{ha}$ with MAE of $\pm 319 \mathrm{~kg} / \mathrm{ha}$, respectively.
\end{abstract}

Keywords: herbaceous cotton, water deficit, semiarid, yield.

Autor de correspondência: Fabrício Daniel dos Santos Silva, fabricio.santos@icat.ufal.br. 


\section{Introdução}

A cultura do algodão herbáceo já foi a mais importante e rentável prática agrícola do Nordeste brasileiro, que já chegou a plantar mais de um milhão de hectares, como na safra 1984/85. Pragas e adversidades climáticas sempre foram fatores limitantes da produção (Almeida et al., 2008). Com o avanço no controle das pragas, o fator climático torna-se o mais importante ator para o sucesso ou fracasso das safras, tendo em vista que a maior parte da produção é sob regime de sequeiro (Azevedo e Maciel, 1993).

Segundo a Associação Brasileira dos produtores de Algodão (ABRAPA, 2020), esta commodity agrícola é produzida por mais de oitenta países, sendo Estados Unidos, Brasil, Índia, Austrália e Uzbequistão os maiores exportadores mundiais da fibra, com o Brasil exportando na última safra 2019/20 mais de 93000 toneladas. Atualmente os Estados do Mato Grosso e Bahia são responsáveis por mais de $82 \%$ da produção nacional (Rosolem, 2007; Carvalho, 2008; Morello et al., 2011). A região Nordeste do Brasil apresenta condições climáticas favoráveis à cotonicultura, haja vista que o algodão necessita de temperaturas ambientais na faixa de 18 a $30^{\circ} \mathrm{C}$, elevadas radiação solar e insolação. Acrescente-se a isto, a existência de cultivares de ciclo (da emergência a primeira colheita) curto (100-120 dias) e médio (130-150 dias), que consomem entre 450 e $700 \mathrm{~mm}$ de água e apresentam potencial de rendimento superior a $3.000 \mathrm{~kg} / \mathrm{ha}$ de algodão em caroço (Azevedo e Silva, 2007).

O algodão é uma das culturas mais adaptadas as condições semiáridas, mas ainda assim, a ocorrência de déficit hídrico com elevada frequência (Silva et al., 2013; Cunha et al., 2015; Alvalá et al., 2017; Martins et al., 2018a; Martins et al., 2018b; Cunha et al., 2018; Marengo et al., 2018; Cunha et al., 2019) e baixa adoção de tecnologias, como a mecanização contribuíram para a redução na área plantada de algodão no semiárido brasileiro (Azevedo et al., 2004).

Uma alternativa recente é a adoção da produção de variedades especiais, como o algodão agroecológico e o algodão colorido (Vidal Neto e Carvalho, 2006; Xiao et al., 2007; Azevedo et al., 2008; Cardoso et al., 2011; Panelas e Silva, 2012). Entre as vantagens para a retomada da produção em larga escala pelos agricultores familiares do semiárido estão a tradição com este tipo de cultivo, o baixo custo de insumos como inseticidas e fungicidas, e principalmente a garantia de mercado na própria região Nordeste, que possui um grande parque têxtil instalado, além do produto não ser perecível sob condições normais de armazenamento (Azevedo et al., 2008).

Diante do exposto, é indispensável para a manutenção e sucesso das safras um sistema ótimo de monitoramento e previsão das safras a partir de dados climáticos, que possa caracterizar os insucessos devido ao excesso ou deficiência hídrica. A estimativa da produtividade agrícola por meio de modelos matemáticos visa simular ou estabelecer relações entre as condições de crescimento das culturas e sua produtividade. Os modelos de produtividade procuram explicar, baseados em processos físicos e fisiológicos, o efeito de uma ou mais variáveis meteorológicas sobre a resposta da planta. Neste contexto, o objetivo desta pesquisa consistiu em calibrar e validar uma adaptação imposta ao modelo clássico proposto por Doorembos e Kassam (1979) visando estimar com mais acurácia a produtividade das safras do algodão herbáceo, não só percentuais, mas também em quilogramas por hectare de área plantada, permitindo a comparação com as estimativas de produtividade do IBGE.

\section{Material e Métodos}

\subsection{Dados e área de estudo}

Os dados de produtividade do algodão herbáceo para a região Nordeste foram disponibilizados pelo Instituto Brasileiro de Geografia e Estatística (IBGE), através de seu Sistema IBGE de Recuperação Automática (SIDRA). Pelo SIDRA, foi possível obter dados da produtividade média da produção, em quilogramas por hectare, das lavouras temporárias do algodão herbáceo, entre 2001 e 2011, em 23 cidades onde coincidiu haver estações meteorológicas convencionais do Instituto Nacional de Meteorologia (INMET). Foram utilizados dados diários de todas as variáveis coletadas nas estações, além da precipitação, necessárias para o cálculo da evapotranspiração potencial pelo método de Penman-Monteith: temperaturas $\left({ }^{\circ} \mathrm{C}\right)$, umidade relativa (\%), insolação (horas), pressão atmosférica (hPa) e velocidade do vento a 10 metros de altura (m/s). A Fig. 1 mostra a distribuição espacial dos 23 municípios com dados de produtividade e meteorológicos, e a Tabela 1 mostra as informações básicas das 23 estações, como código OMM, Estado à qual pertence, nome do município, coordenadas geográficas e altitude.

\subsection{Modelo agrometeorológico de penalização por deficiência hídrica}

O modelo de simulação para produtividade das culturas utilizado segue a base teórica proposta por Doorenbos e Kassan (1979), que estabelece relações entre perdas de produtividade e deficiência hídrica no decorrer das fases fenológicas da cultura. Este modelo é composto por dois módulos: um que estima a produtividade potencial da safra ou produtividade máxima, e outra que estima o decréscimo do potencial de produtividade em função do déficit hídrico. Esse modelo é função da Evapotranspiração Potencial da Cultura $(E T P c)$, da Evapotranspiração Real da Cultura $(E T R c)$ e do coeficiente de penalização por déficit hídrico (ky), mostrados na (Eq. (1)). 


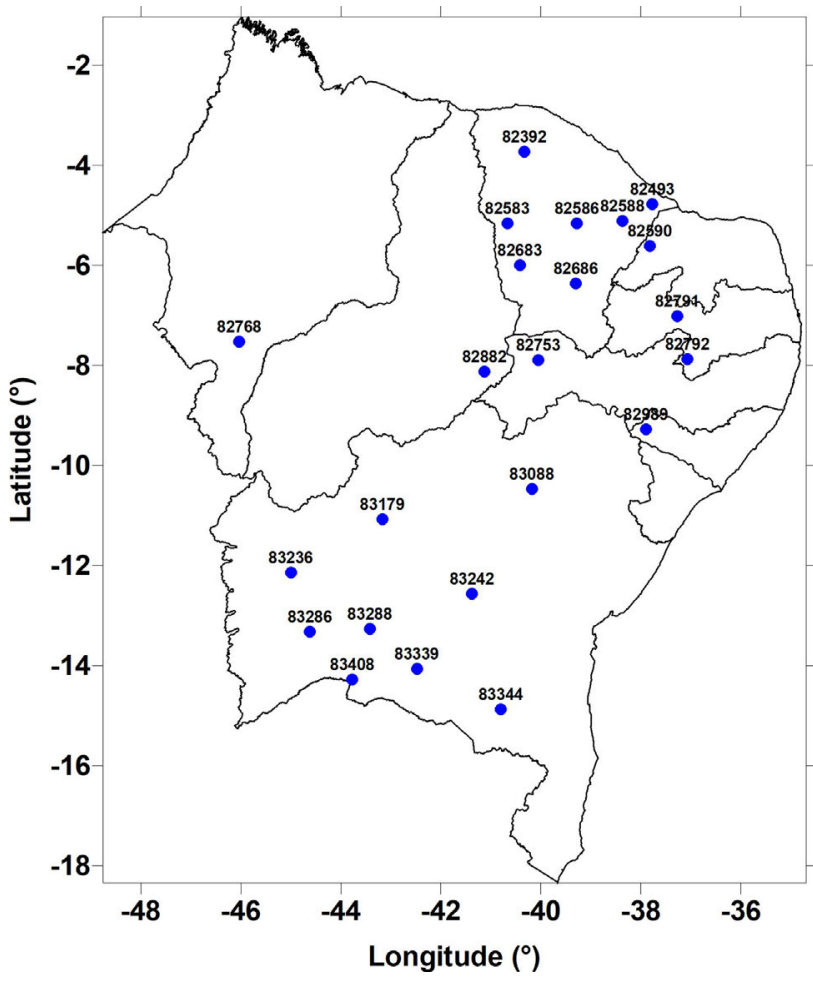

Figura 1 - Distribuição geográfica das 23 estações meteorológicas localizadas em municípios produtores de algodão. Sobre os pontos de estação, o respectivo código da Organização Meteorológica Mundial (OMM).

$$
Y_{d}=\left(1-\frac{E T R_{c}}{E T P_{c}}\right) \times k y \times 100
$$

em que $Y_{d}=$ penalizacao final, ETRc $=$ Evapotranspiração Real da Cultura, ETPc = Evapotranspiração Potencial da Cultura e $k y=$ coeficiente de penalização por déficit hídrico.

No entanto, antes de chegar à etapa final para o cálculo da penalização por déficit hídrico, estágios anteriores tem que ser expostos, e são mostrados na sequência das (Eqs. (2) a (7)). Nos cálculos, o desenvolvimento da cultura do algodão é tabelado para um ciclo da cultura de 150 dias. Este ciclo contém os coeficientes da cultura $(k c)$ e de penalização hídrica $(k y)$, com valores diários (Doorenbos e Kassam, 2000). O cálculo da evapotranspiração da cultura (ETC) depende de um valor para o $k c$, e a evapotranspiração real da cultura $(E T R c)$ depende de um coeficiente de umidade para o solo, o $k s$, calculado conforme o manual de irrigação de Salassier et al. (2008), abaixo.

$$
\begin{gathered}
E T C=E T P \times k c \\
E T R_{c}=k s \times E T P \times k c \\
k s=\frac{\log (A R M+1)}{\log \left(A R M_{c}\right)}
\end{gathered}
$$

\begin{tabular}{|c|c|c|c|c|c|}
\hline Estado & $\begin{array}{l}\text { Código } \\
\text { da } \\
\text { Estação }\end{array}$ & $\begin{array}{l}\text { Nome da } \\
\text { Estação }\end{array}$ & $\begin{array}{l}\text { Latitude } \\
\left(^{\circ}\right)\end{array}$ & $\begin{array}{l}\text { Longitude } \\
\quad\left({ }^{\circ}\right)\end{array}$ & $\begin{array}{l}\text { Altitude } \\
\text { (m) }\end{array}$ \\
\hline Alagoas & 82989 & Água Branca & $-9,28$ & $-37,90$ & 605,3 \\
\hline Bahia & 83088 & $\begin{array}{l}\text { Senhor do } \\
\text { Bonfim }\end{array}$ & $-10,47$ & $-40,18$ & 558,2 \\
\hline Bahia & 83179 & Barra & $-11,08$ & $-43,17$ & 401,6 \\
\hline Bahia & 83236 & Barreiras & $-12,15$ & $-45,00$ & 439,3 \\
\hline Bahia & 83242 & Lençóis & $-12,57$ & $-41,38$ & 438,7 \\
\hline Bahia & 83286 & Correntina & $-13,33$ & $-44,62$ & 549,5 \\
\hline Bahia & 83288 & $\begin{array}{c}\text { Bom Jesus da } \\
\text { Lapa }\end{array}$ & $-13,27$ & $-43,42$ & 440,0 \\
\hline Bahia & 83339 & Caetité & $-14,07$ & $-42,48$ & 882,5 \\
\hline Bahia & 83344 & $\begin{array}{l}\text { Vitória da } \\
\text { Conquista }\end{array}$ & $-14,88$ & $-40,80$ & 874,8 \\
\hline Bahia & 83408 & Carinhanha & $-14,28$ & $-43,77$ & 450,2 \\
\hline Ceará & 82392 & Sobral & $-3,73$ & $-40,33$ & 109,6 \\
\hline Ceará & 82493 & Jaguaruana & $-4,78$ & $-37,77$ & 11,7 \\
\hline Ceará & 82583 & Crateús & $-5,17$ & $-40,67$ & 296,8 \\
\hline Ceará & 82586 & Quixeramobim & $-5,17$ & $-39,28$ & 79,5 \\
\hline Ceará & 82588 & Morada Nova & $-5,12$ & $-38,37$ & 43,6 \\
\hline Ceará & 82683 & Tauá & $-6,00$ & $-40,42$ & 398,8 \\
\hline Ceará & 82686 & Iguatú & $-6,37$ & $-39,30$ & 217,7 \\
\hline Piauí & 82882 & Paulistana & $-8,13$ & $-41,13$ & 374,2 \\
\hline Pernambuco & 82753 & Ouricuri & $-7,90$ & $-40,05$ & 459,3 \\
\hline $\begin{array}{l}\text { Rio Grande } \\
\text { do Norte }\end{array}$ & 82590 & Apodí & $-5,62$ & $-37,82$ & 150,0 \\
\hline Paraíba & 82791 & Patos & $-7,02$ & $-37,27$ & 249,1 \\
\hline Paraíba & 82792 & Monteiro & $-7,88$ & $-37,07$ & 603,7 \\
\hline Maranhão & 82768 & Balsas & $-7,53$ & $-46,03$ & 259,4 \\
\hline
\end{tabular}

Tabela 1 - Estado, código, nome, latitude, longitude e altitude das 23 estações meteorológicas do INMET da região Nordeste onde há produção de algodão.

$$
\begin{gathered}
C A D=\frac{k c}{k c_{\max }} \times C A D_{\max } \\
A R M=A R M_{\text {ant }}-E T R c+\text { Precipitação } \\
A R M_{c}=(1-p) \times \mathrm{CAD}
\end{gathered}
$$

em que $E T P=$ evapotranspiração; $E T P c=$ evapotranspiração da cultura; $C A D=$ capacidade de campo; $A R M=$ armazenamento de água no solo; $A R M c=$ armazenamento crítico (a partir do qual a planta perde produtividade); ARMant $=$ armazenamento anterior; $\mathrm{p}=$ fator de disponibilidade, tabelado pela Food and Agriculture Organization of the United Nations (FAO); $k s=$ coeficiente de umidade; $k c=$ coeficiente de cultura; $k c_{\text {max }}=$ máximo coeficiente de cultura. O $A R M$ nunca pode ser maior do que a CAD.

Diante da formulação acima mostrada, algumas definições são necessárias. A $C A D$ pode ser definida como a quantidade máxima de água que o solo pode armazenar na 
profundidade explorada pelo sistema radicular da planta, variando ao longo do ciclo das culturas em função das características físicas do solo, nas diferentes profundidades, e devido ao crescimento do sistema radicular até atingir a profundidade efetiva. Para a cultura do algodão, a profundidade do sistema radicular pode chegar, em média, a $60 \mathrm{~cm}$.

A ETP é a mudança de estado da água da fase líquida para a gasosa, no caso de solos parcialmente vegetados (Berlato e Molion, 1981). A ETP foi definida por Thornthwaite (1948) como a quantidade de água utilizada por uma extensa área vegetada, em crescimento ativo, sob condições ótimas de umidade do solo. Penman (1956) definiu ETP como "a quantidade de água utilizada na unidade de tempo por uma cultura de porte baixo e verde, cobrindo totalmente a superfície, com altura uniforme e sem deficiência hídrica". Independente das definições, em 1990 a FAO recomendou o método de Penman-Monteith como padrão na estimativa da ETP (Monteith, 1973; Smith, 1991; Silva et al., 2018).

$\mathrm{O}$ cálculo de ETP diária utiliza dados de temperatura máxima e mínima do ar, umidade relativa do ar, insolação e velocidade do vento medida a dois metros de altura, segundo a (Eq. (8)). Os procedimentos detalhados para obtenção dos parâmetros desta equação são encontrados em Pereira et al. (1997) e Allen et al. (1998).

$$
E T P=\frac{\delta}{\delta+\gamma^{*}}\left(R_{n}-G\right) \frac{1}{\lambda}+\frac{\gamma}{\delta+\gamma^{*}} \frac{900}{T+273}\left(e_{s}-e_{a}\right)
$$

em que ETP é a evapotranspiração diária de referência $(\mathrm{mm}) ; \lambda$ é o calor latente de vaporização $\left(\mathrm{MJ} \mathrm{kg}^{-1}\right) ; d$ é a inclinação da curva da pressão de vapor saturado versus temperatura $\left(\mathrm{k} \mathrm{PaK}^{-1}\right) ; R n$ é o saldo de radiação $\left(\mathrm{MJ} \mathrm{m}^{-2}\right.$ $\left.\mathrm{dia}^{-1}\right) ; G$ é o fluxo de calor no solo $\left(\mathrm{MJ} \mathrm{m}^{-2} \mathrm{dia}^{-1}\right)$; es é a pressão de vapor saturado do ar (k Pa); ea é a pressão de vapor do ar na altura $\mathrm{z}(\mathrm{k} \mathrm{Pa}) ; T$ é a temperatura do ar na altura $\mathrm{z}\left({ }^{\circ} \mathrm{C}\right) ; \lambda *$ é o coeficiente psicrométrico modificado $\left(\mathrm{k} \mathrm{Pa} \mathrm{K}^{-1}\right)=\lambda\left(1+0,33 U_{2}\right) ; U_{2}$ é a Velocidade do vento medida a 2 metros de altura $\left(\mathrm{m} \mathrm{s}^{-1}\right) ; 900$ é uma Constante $\left(\mathrm{k} \mathrm{J}^{-1} \mathrm{~kg}{ }^{\circ} \mathrm{K}\right)$.

\subsection{Calibração do modelo agrometeorológico}

O modelo agrometeorológico proposto foi calibrado para o período 2001-2011, com dados de produtividade do algodão herbáceo, em $\mathrm{kg} / \mathrm{ha}$, para a área plantada de 23 municípios localizados na região semiárida do NEB, de acordo com as épocas de semeadura indicadas pela Companhia Nacional de Abastecimento (CONAB), do Governo Federal.

O primeiro passo, de acordo com Monteiro et al. (2013; 2017), foi obter, dos dados de produtividade, a produtividade máxima chamada de Produtividade Máxima Tecnológica (PMT), estimada a partir de um ajuste estatístico baseado na série de dados das Produtividades Reais Observadas $(P R O)$. Uma equação linear de primeiro grau $(Y=a x+b)$, dos dados observados versus o tempo (ano) deve ser obtida, onde a linha reta indica a tendência da produtividade ao longo dos anos (TPA). Os desvios ao longo da linha de tendência são causados por fatores climáticos, de disponibilidade hídrica e de manejo da cultura. A segunda etapa consiste na determinação de um fator de correção que, quando multiplicado pela tendência da produtividade observada (TPA), elevaria os valores de produtividade em uma situação sem restrição de água. Esse fator de correção foi nomeado coeficiente de produtividade máxima tecnológica $(\Delta p)$. Logo, a $P M T$ foi calculada para cada ano "n", em função da produtividade real ajustada multiplicada por $\Delta p: P M T=T P A \times \Delta p$.

Desta forma, $\Delta p$ foi ajustado de uma regressão linear sem tendência e consiste em um dos parâmetros calibrados no modelo. Os valores dos coeficientes de cultura $(k c)$ e de déficit hídrico $(k y)$, apresentados por Doorembos e Kassam (2000) para o algodão herbáceo, de acordo com suas fases fenológicas, foram adaptados para a escala diária, facilitando sua posterior obtenção para a escala decendial, como mostra a Tabela 2.

A Produtividade Real $(P R)$ foi estimada a partir da relação entre o déficit relativo de água [1- $(E T R c / E T P c)]$, e as perdas de produtividade relativa, ponderada pelo fator de déficit hídrico $(k y)$, para cada fase fenológica:

$$
Y d=\frac{P R}{P M T}=\left[1-\left(\frac{E T R c}{E T P c}\right)\right] \times k y \times 100
$$

Neste modelo, uma alteração importante é encontrar, para qualquer estação meteorológica, de acordo com a sua classe de produtividade (muito baixa, baixa, média ou alta), um parâmetro empírico denominado "fator de déficit hídrico ajustado", ou " $k y *$ ", que resulta em maior ou menor resposta ao estresse hídrico. Outra diferença é a utilização da PMT. Com tais mudanças, o modelo final apresentará a seguinte equação (Eq. (10)):

\begin{tabular}{|c|c|c|c|c|c|c|c|c|c|c|c|c|c|c|c|}
\hline \multicolumn{16}{|c|}{ Coeficientes para o Algodão } \\
\hline Decêndios & 1 & 2 & 3 & 4 & 5 & 6 & 7 & 8 & 9 & 10 & 11 & 12 & 13 & 14 & 15 \\
\hline Kc & 0,5 & 0,5 & 0,56 & 0,7 & 0,83 & 0,94 & 1,02 & 1,07 & 1,09 & 1,09 & 1,08 & 1,04 & 0,96 & 0,86 & 0,84 \\
\hline Ky & 0,2 & 0,2 & 0,2 & 0,2 & 0,26 & 0,42 & 0,5 & 0,48 & 0,35 & 0,17 & 0,05 & 0,01 & 0 & 0 & 0 \\
\hline
\end{tabular}

Tabela 2 - Valores de Kc e Ky para o Algodão. 


$$
\frac{P R}{P M T}=\left[1-\left(\frac{E T R c}{E T P c}\right)\right] \times k y \times k y^{*} \times 100
$$

em que, nesta equação, a $P M T$ é usada como referência inicial para começar o procedimento de cálculo das perdas, para cada estação.

O processo de parametrização consiste no ajuste do coeficiente de produtividade máxima tecnológica $(\Delta p)$, e do " $k y^{*}$ ", a fim de maximizar a correlação entre as produtividades reais observadas e estimadas, e também para minimizar o erro médio absoluto (EMA) entre eles. Neste processo, $k y^{*}$ começa com valor igual a 1 , sendo acrescido ou reduzido de acordo com o ganho ou perda na correlação e no EMA entre as produtividades observadas e estimadas. O EMA é caracterizado pela média do módulo dos erros absolutos das estimativas, sendo dado por:

$$
E M A=\frac{1}{n} \sum_{i=1}^{n}\left|P e_{i}-P o_{i}\right|
$$

onde $P e_{i}$ é a produtividade final estimada em um ano $i$ e $P o_{i}$ é a produtividade real observada pelo IBGE no ano $i$, e $n$ é o número de comparações.

No modelo da Eq. (10), a ETRc é calculada pelo balanço hídrico sequencial, onde são computados as mudanças diárias na umidade do solo, de acordo com o método de Thornthwaite (1948).

A validação do modelo parametrizado se dará pela comparação das produtividades médias obtidas, em $\mathrm{kg} / \mathrm{ha}$ no período 2001-2011, estimada pelo IBGE e simuladas pelo modelo, além do EMA e do erro médio relativo dado pela divisão do $E M A$ pela $P M O$, e do coeficiente de correlação, que mede a força da relação linear entre observações $(o)$ e simulações $(s)$ e é definido como a covariância das duas variáveis, observadas e simuladas, dividida pelo produto de seus desvios-padrão (Eq. (12)):

$$
r_{o, s}=\frac{\operatorname{Cov}(o, s)}{\sigma_{o}}
$$

Para garantir que o valor de $r$ realmente exprima a concordância entre observações e simulações, usou-se o teste paramétrico t-Student (Huang e Paes, 2009; Menezes, 2010) para avaliar o grau de relação entre as variáveis em questão. Uma premissa para o uso do teste é que o tamanho da amostra, $N$, da qual obtém-se o valor do coeficiente de correlação, $r$, seja igual ou superior a 6 , então o valor de $t$ é dado por:

$$
t=\frac{r}{\sqrt{\frac{\left(1-r^{2}\right)}{(N-2)}}}
$$

A Eq. (14) é uma distribuição para $t$ com $N$-2 graus de liberdade. A aplicação desta fórmula para qualquer valor de $r$ e de $N$ vai testar a hipótese nula de que o valor observado vem de uma população em que não existe correlação sig- nificativa entre os dados. Uma vez obtido o valor de $t$, pode-se extrair o coeficiente de correlação critico $\left(r_{c}\right)$, que é um valor para a qual se aceita ou não a hipótese estatística que existe correlação entre os dados simulados e observados, $r_{c}$ é dado por:

$$
r_{c}=\sqrt{\frac{t^{2}}{(N-2)+t^{2}}}
$$

\section{Resultados e Discussão}

De acordo com os dados de produtividade anual do algodão herbáceo disponibilizados pelo IBGE, entre 2001 e 2011, existe grande variabilidade entre os 23 municípios pesquisados. A produtividade média anual nestes 11 anos varia de $259 \mathrm{~kg} / \mathrm{ha}$ em Água Branca (AL), até $3652 \mathrm{~kg} / \mathrm{ha}$ em Bom Jesus da Lapa (BA), ambas as cidades localizadas no semiárido nordestino. A maioria dos municípios apresenta produtividades medias inferiores a $1000 \mathrm{~kg} / \mathrm{ha}$. A Fig. 2 mostra esta variação, ou seja, as produtividades médias em cada município segundo o cenário climático atual a que está submetida a cultura.

Esta variabilidade reflete como dois importantes fatores influenciam a produtividade do algodão entre os municípios: o grau de vulnerabilidade ao estresse hídrico e a influência tecnológica. Por isso, foram calculados o coeficiente de rendimento máximo tecnológico $(\Delta p)$ e o fator de déficit hídrico ajustado $\left(k y^{*}\right)$ para cada município, durante o processo de calibração do modelo de produtividade. Estes parâmetros permitiram simular, com mais precisão, a produtividade em cada ano.

Primeiro, é obtida uma estimativa da produtividade, para cada município, sem levar em consideração o rendimento máximo tecnológico $(\Delta p)$ e o fator de déficit hídrico ajustado $(k y *)$, pela formulação clássica proposta por Doorenbos e Pruitt (1997), cuja penalização depende apenas da relação entre ETRc e ETPc. Em seguida, ao se calcular a produtividade potencial tecnológica, com a correção das tendências de rendimento ao longo do tempo, a precisão das estimativas tanto dos baixos quanto dos altos rendimentos é melhorada e podem-se aproximar os valores de perda percentual da produtividade aos valores observados pelo IBGE, calibrando o modelo e encontrando os valores de $\Delta p$ e $k y *$. Em alguns casos, onde o fator de estresse hídrico não se torna muito relevante, o valor de $k y *$ continua igual a 1 .

Para exemplificar a importância destes parâmetros na simulação da produtividade, os gráficos da Fig. 3 mostram as curvas, entre 2001 e 2011, da estimativa de produtividade versus a produtividade observada nos municípios de Carinhanha (BA), antes (a) e depois (b) do processo de calibração, e em Crateús (CE), antes (c) e depois (d) do processo de calibração.

A Tabela 3 mostra os valores da produtividade média prevista e observada, os valores do $\Delta p$ e do $k y^{*}$ encontrados para cada município a fim de obter a maior correla- 


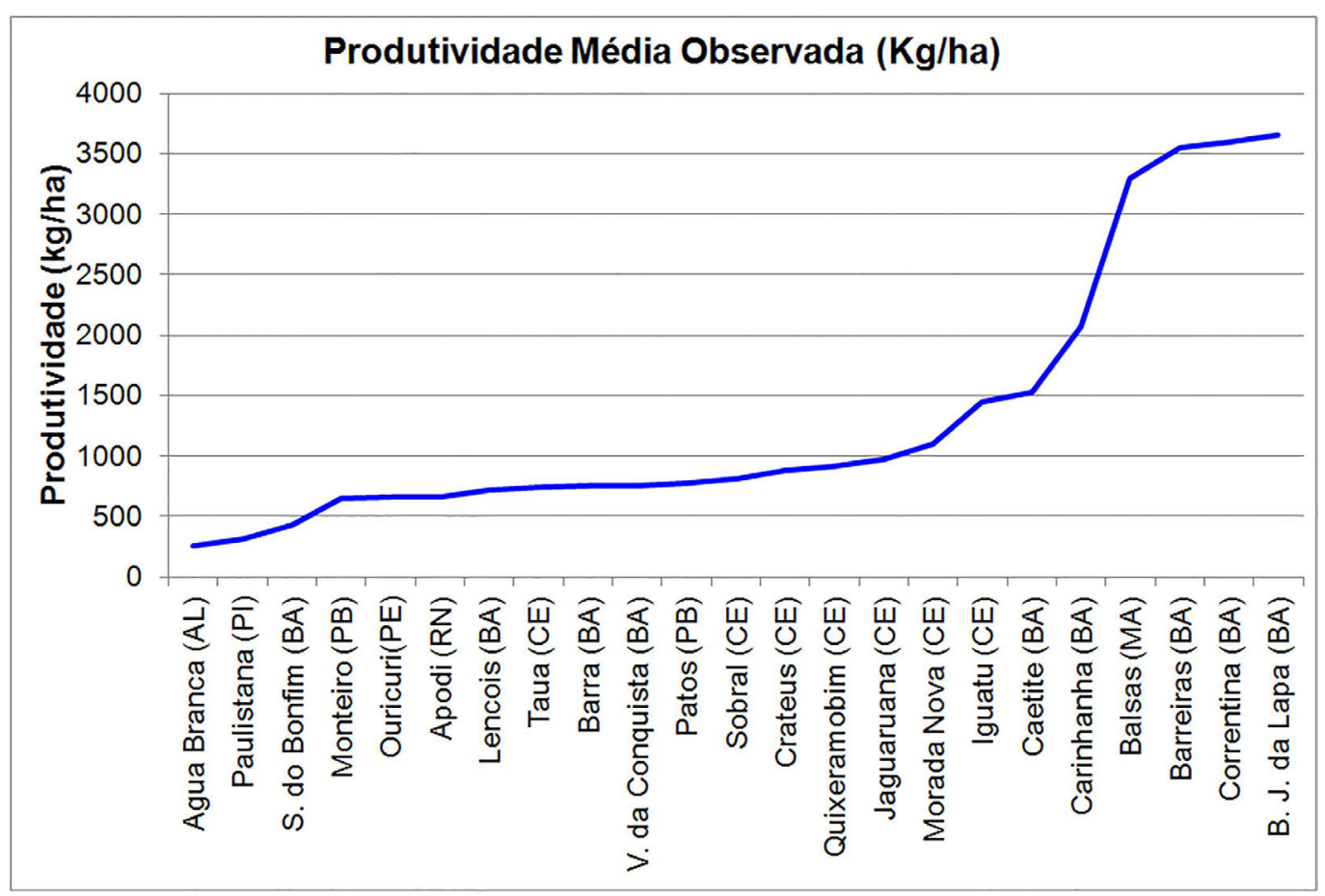

Figura 2 - Produtividade média do algodão herbáceo (kg/ha) em cada município, entre 2001 e 2011, fornecido pelo IBGE.

(a) Produtividades Observadas e Estimadas do Algodao Herbaceo

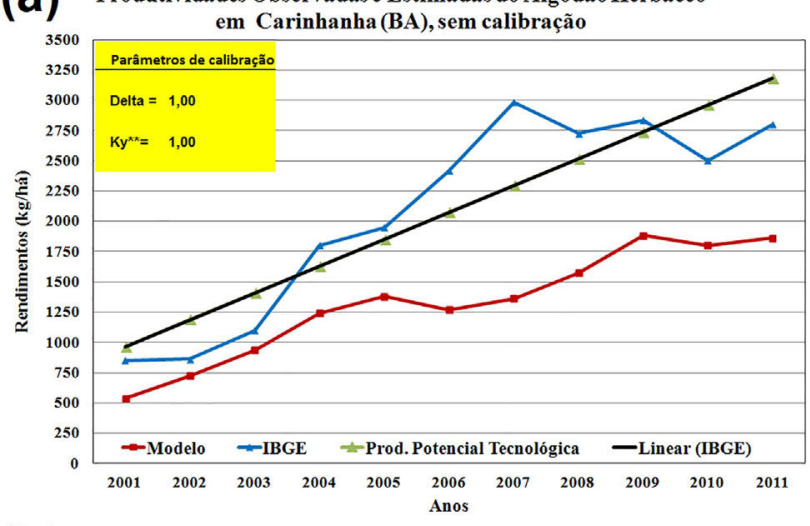

(b) Produtividades Observadas e Estimadas do Algodao Herbaceo

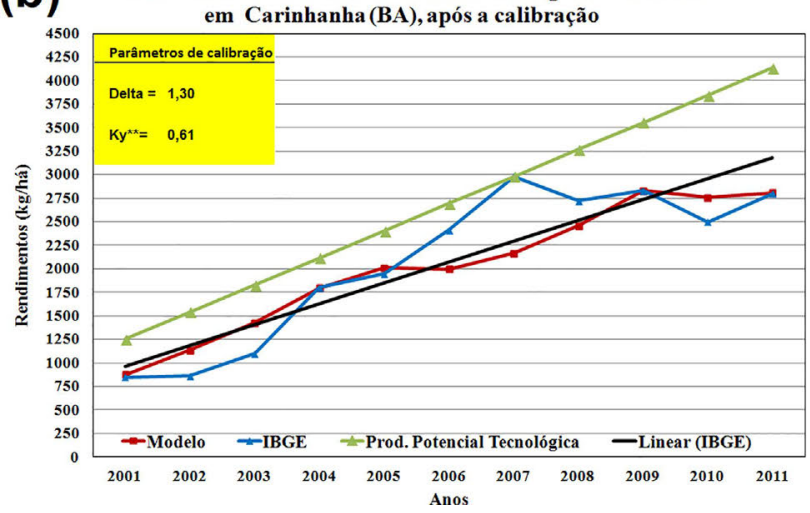

(C) Produtividades Observadas e Estimadas do Algodao Herbaceo

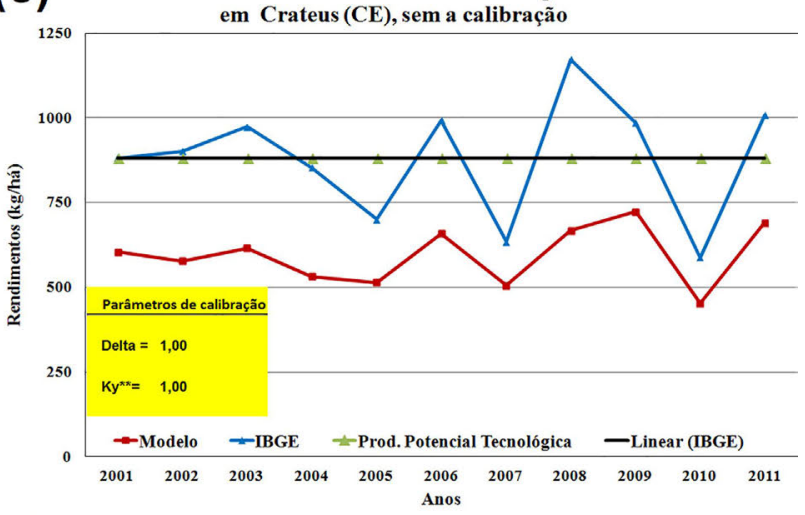

(d) Produtividades Observadas e Estimadas do Algodao Herbaceo

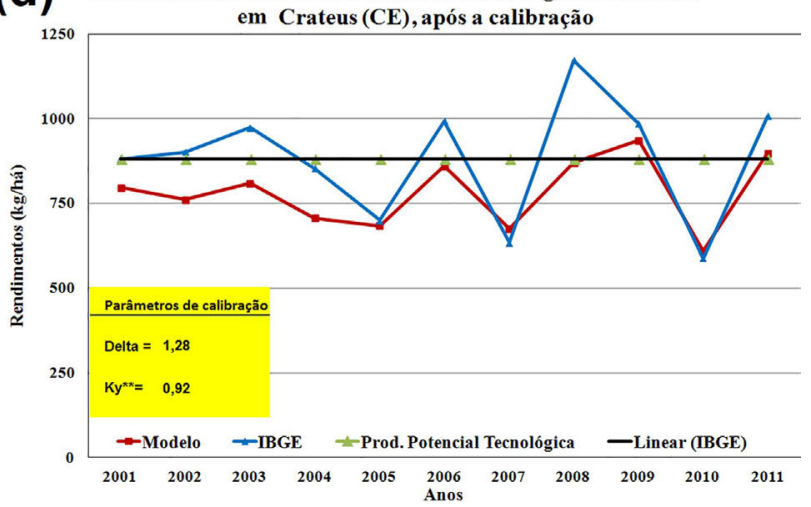

Figura 3 - Produtividades observadas e simuladas do algodão herbáceo nas cidades de Carinhanha (a e b) e Crateús (c e d), antes e depois da calibração do modelo agrometeorológico. 
Tabela 3 - Produtividade média observada e simulada, erro médio absoluto (EMA) e relativo (EMR), coeficiente de produtividade máxima tecnológica $(\Delta \mathrm{p})$, fator de déficit hídrico ajustado $\left(k y^{*}\right)$, e correlação $(r)$ entre simulações e observações. Valores de $r$ acima de 0,60 estão destacados em cinza.

\begin{tabular}{|c|c|c|c|c|c|c|c|}
\hline Estação & Produtividade Média Observada $(\mathrm{kg} / \mathrm{ha})$ & Produtividade Média Simulada(kg/ha) & EMA (kg/ha) & $\operatorname{EMR}(\%)$ & $\Delta \mathrm{p}$ & $K y^{*}$ & $r$ \\
\hline Agua Branca (AL) & 259 & 288 & 41 & 15,7 & 1,25 & 1,00 & 0,63 \\
\hline Apodi (RN) & 664 & 716 & 76 & 10,4 & 1,27 & 0,58 & 0,68 \\
\hline Balsas (MA) & 3292 & 3405 & 193 & 5,3 & 1,20 & 1,00 & 0,63 \\
\hline Barra (BA) & 754 & 808 & 267 & 32,7 & 1,71 & 0,96 & 0,37 \\
\hline Barreiras (BA) & 3548 & 3721 & 324 & 9,1 & 1,22 & 0,53 & $0,78^{*}$ \\
\hline B. J. da Lapa (BA) & 3652 & 3959 & 582 & 15,9 & 1,32 & 0,53 & 0,69 \\
\hline Caetite (BA) & 1528 & 1585 & 229 & 15 & 1,59 & 1,00 & 0,65 \\
\hline Carinhanha (BA) & 2074 & 2024 & 224 & 10,8 & 1,30 & 0,61 & $0,92^{* *}$ \\
\hline Correntina (BA) & 3601 & 3675 & 270 & 7,5 & 1,18 & 0,42 & 0,57 \\
\hline Lencois (BA) & 716 & 972 & 395 & 55,1 & 1,95 & 1,00 & 0,22 \\
\hline Monteiro (PB) & 647 & 686 & 112 & 17,3 & 1,30 & 0,47 & 0,56 \\
\hline Ouricuri(PE) & 661 & 640 & 41 & 6,2 & 1,23 & 0,46 & 0,75 \\
\hline Patos (PB) & 781 & 750 & 231 & 29,6 & 1,54 & 1,00 & 0,54 \\
\hline S. do Bonfim (BA) & 428 & 436 & 15 & 3,4 & 1,07 & 0,17 & $0,79^{*}$ \\
\hline V. Conquista (BA) & 756 & 948 & 308 & 40,8 & 1,82 & 1,00 & 0,44 \\
\hline Crateus (CE) & 881 & 783 & 109 & 12,4 & 1,28 & 0,92 & $0,89^{*}$ \\
\hline Jaguaruana (CE) & 970 & 1015 & 111 & 11,4 & 1,52 & 1,00 & $0,84^{*}$ \\
\hline Iguatu (CE) & 1448 & 1540 & 250 & 17,2 & 1,46 & 1,00 & $0,78^{*}$ \\
\hline Taua (CE) & 746 & 726 & 190 & 25,5 & 1,54 & 1,00 & 0,63 \\
\hline Sobral (CE) & 806 & 824 & 99 & 12,3 & 1,28 & 0,92 & $0,80^{\circ}$ \\
\hline Quixeramobim (CE) & 909 & 900 & 124 & 13,7 & 1,45 & 0,91 & 0,60 \\
\hline Morada Nova (CE) & 1099 & 1075 & 115 & 10,4 & 1,22 & 0,56 & 0,73 \\
\hline Paulistana (PI) & 312 & 359 & 111 & 35,7 & 1,90 & 0,99 & 0,75 \\
\hline
\end{tabular}

Valores estatisticamente significantes ao nível de 95\% de confiança;

*** Valores estatisticamente significantes ao nível de 99\% de confiança.

ção e o menor erro absoluto possível. Analisando os valores de $r$, percebe-se que os ajustes impostos ao modelo para estimativa da produtividade foram bem sucedidos. Para o tamanho da amostra, ou seja, N=11 anos de comparações, o teste de significância estatística aponta como valor de correlação crítica $r_{c}=0,92$. Qualquer valor de $r$ maior ou igual a 0,92 é estatisticamente significativo ao nível de $99 \%$ de confiança. Como este valor é muito alto e difícil de alcançar para apenas $\mathrm{N}=11$ comparações, adotase o nível de confiança de $95 \%$, cujo $r_{c}=0,78$. Dos 23 locais estudados, 6 apresentaram correlações estatisticamente significantes, mais da metade (13 estações) apresentaram valores de $r$ entre 0,6 e 0,8 , ou fortes correlações, e em cinco os valores de $r$ ficaram entre 0,8 e 1,0 , indicando ótimas correlações. Nenhuma estação apresentou $r$ inferior a 0,2, duas estações apresentaram $r$ entre 0,2 e 0,4 e por fim quatro estações apresentaram $r$ entre 0,4 e 0,6 , ou correlações moderadas.

Analisando as estações de Barra e Lençóis (BA), que apresentaram baixas correlações, entre 0,2 e 0,4 , observase que as mesmas também apresentaram os maiores erros relativos, ano a ano, culminando em um erro médio relativo de 55,1\% em Lençóis e de 32,7\% em Barra. Isso sig- nifica que entre 2001 e 2011, a produtividade estimada pelo modelo apresentou diferenças significativas das observadas nestas cidades, muitas vezes em sentidos opostos, caracterizando as baixas correlações. Esses erros sistemáticos poderiam ser amenizados por meio de uma re-calibração do modelo agrometeorológico, mas, para tanto, necessitaríamos de séries de produtividades observadas bem maiores do que as que se foi possível trabalhar, permitindo uma melhor análise da variabilidade interanual destas produtividades. Diante deste pequeno número de casos em que o modelo não se saiu bem em suas estimativas, pode-se inferir que as fontes de erros podem estar relacionadas com eventuais alterações das práticas agrícolas e de gestão, que incluem os genótipos utilizados, manejo do solo, adubação, doenças e surtos de pragas, etc. Estes são fatores não computados pelo modelo, devido à impossibilidade de examiná-los, sistematicamente, nas amplas áreas a serem consideradas. Portanto, variações imprevisíveis podem ocorrer e prejudicar o desempenho do modelo.

Em contrapartida, 16 das 23 estações apresentaram correlações superiores a 0,6 . Nestes casos, foram observados os menores erros relativos entre os valores simulados 


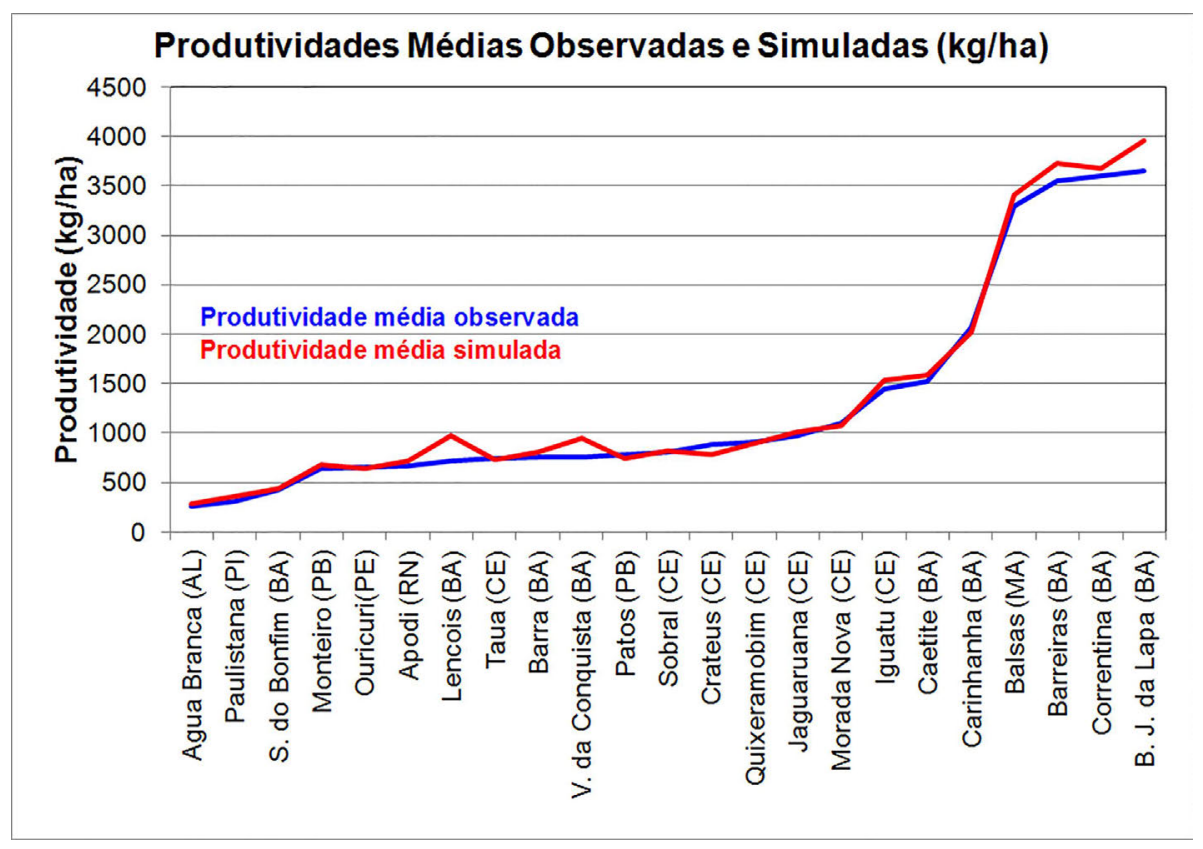

Figura 4 - Produtividades médias do algodão herbáceo (kg/ha) em cada município, entre 2001 e 2011, observadas e estimadas pelo modelo agrometeorológico.

e observados. Analisando os casos onde a correlação foi igual ou superior a 0,80 , nas localidades de Carinhanha (BA), Crateús, Jaguaruana e Sobral (CE), o erro médio absoluto ficou em torno de $\pm 150 \mathrm{~kg} / \mathrm{ha}$, ou um erro médio relativo inferior a $15 \%$, entre rendimentos estimados e observados.

Vale ressaltar que, para a maior parte dos municípios analisados, o erro simples absoluto (ESA), quando se analisa apenas a diferença das médias simuladas e observadas, foi positivo, evidenciando uma tendência do modelo em superestimar a produtividade média observada. Para municípios com produtividades até $500 \mathrm{~kg} / \mathrm{ha} \mathrm{o} E S A$ foi de aproximadamente $28 \mathrm{~kg} / \mathrm{ha}$, nos municípios com produtividades de 500 a $1000 \mathrm{~kg} / \mathrm{ha}$ o $E S A$ foi de aproximadamente $40 \mathrm{~kg} / \mathrm{ha}$, nos municípios com produtividades de 1000 a $2000 \mathrm{~kg} / \mathrm{ha}$ o $E S A$ foi de aproximadamente $42 \mathrm{~kg} / \mathrm{ha}$, e por fim em municípios com produtividades superiores a $2000 \mathrm{~kg} / \mathrm{ha}$ o ESA foi de aproximadamente $123 \mathrm{~kg} / \mathrm{ha}$.

Por fim, a Fig. 4 mostra a produtividade média observada versus a produtividade média simulada em cada município. Vale salientar que o cálculo da produtividade para cada município é função inicialmente do cálculo da penalização por déficit hídrico, que indica o percentual de perda da produtividade em três datas estabelecidas e determinadas pelo Governo Federal para a semeadura no campo, correspondentes ao calendário agrícola. Para cada uma das três datas, é calculada a penalização, assumindose que, em média, $25 \%$ da produção é plantada na primeira data, $50 \%$ da produção é plantada na segunda data e $25 \%$ da produção é plantada na terceira data.

\section{Conclusões}

A calibração do modelo agrometeorológico permitiu estimar a produtividade do algodão, em cada uma das 23 estações entre 2001 e 2011, com boa concordância em relação as produtividades observadas pelo IBGE. A variabilidade anual, que acarretou em baixos coeficientes de correlação para algumas estações, incorre de um problema comum em qualquer tipo de modelagem, que é a capacidade de simular extremos. Desta forma, a média dos 11 anos de observações e simulações, para cada município estudado, mostrou a habilidade do modelo em simular os valores médios de produtividade, tanto nos municípios de produtividade muito baixa até os de alta produtividade, conseguindo acompanhar a curva de ascendência imposta pela produtividade potencial tecnológica.

\section{Agradecimentos}

Os autores agradecem a Coordenação de Aperfeiçoamento de Pessoal de Nível Superior (CAPES) pelo apoio financeiro concedido durante e para a concepção deste estudo.

\section{Referências}

ABRAPA: Algodão no Mundo, 2020 (https://www.abrapa.com. br/Paginas/dados/algodao-no-mundo.aspx). Acessado em 27 de junho de 2020.

ALLEN, R.G., PEREIRA, L.S., RAES, D., SMITH, M. Crop Evapotranspiration: Guidelines for Computing Crop Water Requirements. FAO Irrigation and Drainage Paper 56, Rome, 1998. 
ALMEIDA, R.P.; SILVA, C.A.D.; RAMALHO, F.S. Manejo integrado de pragas do algodoeiro no Brasil. In: Beltrão N.E.M.; Azevedo D.M.P. (eds.) O Agronegócio do Algodão no Brasil. Brasília: Embrapa Informação Tecnológica, p. 1034-1098, 2008.

ALVALA, R.C.S.; CUNHA, A.P.M.A.; BRITO, S.S.B.; SELUCHI, M.E.; MARENGO, J.A.; MORAES, O.L.L.; CARVALHO, M.A. Drought monitoring in the Brazilian Semiarid region. Anais da Academia Brasileira de Ciências, v. 91, n. 1, p. 1-15 2017.

AZEVEDO, P.V.; MACIEL, G.F. Estação de cultivo e época de semeadura para o algodão herbáceo na região de Sousa-PB. Revista Brasileira de Agrometeorologia, v. 1, n. 1, p. 8185, 1993.

AZEVEDO, P.V.; SILVA, F.D.S.; BEZERRA, J.R.C. Zoneamento da época de semeadura do algodoeiro herbáceo no estado da Paraíba. Revista Brasileira de Agrometeorologia, v. 12, n. 2, p. 379-386, 2004.

AZEVEDO, P.V.; SILVA, F.D.S. Risco climático para o cultivo do algodoeiro na região nordeste do Brasil. Revista Brasileira de Meteorologia, v. 22, n. 3,p. 408-416, 2007.

AZEVEDO, D.M.P.; BELTRÃO, N.E.M.; FERREIRA, A.C.B.; LEÃO, A.B.; CARDOSO, G.D.; VIEIRA, D.J.; NÓBREGA, L.B. Manejo de plantas daninhas. In: BELTRÃO N.E.M.; AZEVEDO D.M.P. (eds.) O Agronegócio do Algodão no Brasil. Brasília: Embrapa Informação Tecnológica, p. 271-298, 2008.

BERLATO, M.A.; MOLION, L.C.B. Evaporação e Evapotranspiração. Porto Alegre: IPAGRO, 95 p, 1981. (IPAGRO. Boletim Técnico, 7).

CARDOSO, G.D.; ALVES, P.L.C.A.; SEVERINO, L.S.; VALE, L.S. Critical periods of weed control in naturally green colored cotton BRS Verde. Industrial Crops and Products, v. 34, n. 1, p. 1198-1202, 2011.

CARVALHO, L.P. Contribuição do melhoramento ao cultivo do algodão. In: BELTRÃO N.E.M.; AZEVEDO D.M.P. (eds.) O Agronegócio do Algodão no Brasil. Brasília: Embrapa Informação Tecnológica, p. 271-298, 2008.

CUNHA, A.P.M.A.; ALVALÁ, R.C.S.; NOBRE, C.A.; CARVALHO, M.A. Monitoring vegetative drought dynamics in the Brazilian semiarid Region. Agricultural and Forest Meteorology, v. 214, p. 494-505, 2015.

CUNHA, A.P.M.A.; TOMASELLA, J.; RIBEIRO-NETO, G.G.; BROWN, M.; GARCIA, S.R.; BRITO, S.B.; CARVALHO, M.A. Changes in the spatial-temporal patterns of droughts in the Brazilian Northeast. Atmospheric Science Letters, v. 19, n. 10, e855, 2018.

CUNHA, A.P.M.A.; ZERI, M.; LEAL, K.D.; COSTA, L.; CUARTAS, L.A.; MARENGO, J.A.; TOMASELLA, J.; VIEIRA, R.M.; BARBOSA, A.A.; CUNNINGHAM, C.; GARCIA, J.V.C.; BROEDEL, E.; ALVALÁ, R.; RIBEIRO-NETO, G. Extreme Drought Events over Brazil from 2011 to 2019. Atmosphere, v. 10, n. 11, p. 642, 2019.

DOORENBOS, J; KASSAM, A.H. Yield Response to Water. Roma, Irrigation and Drainage Paper 33, FAO, 179p, 1979.

DOORENBOS, J; PRUITT, W.O. Necessidades hídricas das culturas. Tradução de GHEYI, H.R.; METRI, J.E.C.; DAMASCENO, F.A.V. Campina Grande: UFPB (Estudos FAO: Irrigação e Drenagem, 24), 1997.
DOOREnBOS, J; KASSAM, A.H. Efeito da Água no Rendimento das Culturas. Tradução de GHEYI, H.R.; SOUSA, A.A.; DAMASCENO, J.F. 2. ed. Campina Grande: UFPB, 221p, 2000. (Estudos FAO: Irrigação e Drenagem, 33).

HUANG, G.; PAES, A.T. Posso usar o teste t-Student quando preciso comparar três ou mais grupo? Einstein: Educação Continuada em Saúde, v. 7, n. 2, p. 63-64, 2009.

MARENGO, J.A.; ALVES, L.M.; ALVALA, R.C.C.; CUNHA, A.P.M.A.; BRITO, S.B.; MORAES, O.L.L. Climatic characteristics of the 2010-2016 drought in the semiarid Northeast Brazil region. Anais da Academia Brasileira de Ciências, v. 90, n. 2, p. 1973-1985, 2018.

MARTINS, E.S.P.R.; COELHO, C.A.S.; HAARSMA, R.; OTTO, F.E.L.; KING, A.D.; VAN OLDENBORGH, G.J.; KEW, S.; PHILIP, S.; JÚNIOR, F.C.V.; CULLEN, H. A multimethod attribution analysis of the prolonged northeast Brazil hydrometeorological drought (2012-16). Bulletin of the American Meteorological Society, v. 99, n. 1, p. 6569, 2018a.

MARTINS, M.A.; TOMASELLA, J.; RODRIGUEZ, D.A.; ALVALÁ, R.C.; GIAROLLA, A.; GAROFOLO, L.L.; JÚNIOR, J.L.S.; PAOLICCHI, L.T.; PINTO, G.L. Improving drought management in the Brazilian semiarid through crop forecasting. Agricultural Systems, v. 160, p. 21-30, $2018 b$.

MENEZES, H.E.A. Influência da Zona de Convergência Secundária do Atlântico Sul sobre a Ocorrência de Precipitação no Leste do Nordeste Brasileiro. Tese de Doutorado - UFCG, Campina Grande-PB, 103p. 2010.

MONTEIRO, J.E.B.A.; AZEVEDO, L.C.; ASSAD, E.D.; SENTELHAS, P.C. Rice yield estimation based on weather conditions and on technological level of production systems in Brazil. Pesquisa Agropecuária Brasileira, v. 48, n. 2, p. 123-131, 2013.

MONTEIRO, J.E.B.A.; ASSAD, E.D.; SENTELHAS, P.C.; AZEVEDO, L.C. Modeling of corn yield in Brazil as a function of meteorological conditions and technological level. Pesquisa Agropecuária Brasileira, v. 52, n. 3, p. 137-148, 2017.

MONTEITH, J.L. Principles of Environmental Physics. London, Edward Arnold, 241p, 1973.

MORELLO, C.L.; SILVA, C.A.D.; SUASSUNA, N.D.; SILVA, O.R.R.F. Cultivares de Algodoeiro para o Cerrado. Campina Grande: Embrapa Algodão, 2011.

PANELAS, O.; SILVA J.S. Outra Visão do Sertão. Campina Grande: Instituto Nacional do Semiárido, 39p, 2012.

PEREIRA, A.R.; VILLA NOVA, N.A.; SEDIYAMA, G.C. Evapo(transpi)ração. Piracicaba: FEALQ, 183p, 1997.

PENMAN, H.L. Evaporation: Introduction survey. Netherlands Journal of Agricultural Science. v. 4, n. 1, p. 9-29, 1956.

ROSOLEM, C.A. Fatores fisiológicos que afetam a produtividade do algodoeiro. In: FARIAS, F.J.C.; RODRIGUES, S.M.M.; LAMAS, F.M. (Ed.). Tecnologia para o Algodoeiro no Cerrado do Mato Grosso. Campina Grande: Embrapa Algodão, p. 13-23, 2007.

SALASSIER, B.; SOARES, A.A.; MANTOVANI, E.C. Manual de Irrigação. Editora UFV, 625p, 8.ed, 2008.

SILVA, V.B.S.; KOUSKY, V.E.; SILVA, F.D.S.; SALVADOR, M.A.; ARAVEQUIA, J.A. The 2012 severe drought over 
Northeast Brazil. Bulletin of the American Meteorological Society, v. 94, S240, 2013.

SILVA, F.D.S.; COSTA, R.L.; ANTONIO, M.A.V.; AFONSO, E.O.; SANTOS, D.M.; MATEUS, N.P.A.; ANTONIO, J.F. Tendências observadas da evapotranspiração potencial no estado de Alagoas (1961-2016). Revista Brasileira de Geografia Física, v. 11, n. 1, p. 28-43, 2018.

SMITH, M. Report on the Expert Consultations on Revision of FAO Methodologies for Crop Water Requirements. Rome: FAO, 45p, 1991.

THORNTWAITE, G.W. An approach toward a rational classification of climate. Geographycal Review, New York, v. 38, n. 1, p. 55-94, 1948.
VIDAL NETO, F.C.; CARVALHO, L.P. Cerrado e semiárido requerem cultivares diferenciadas. Visão Agrícola, v. 3, n. 6, p. 32-34, 2006.

XIAO, Y-H.; ZHANG, Z-S.; YIN, M-H.; LUO, M.; LI, X-B.; HOU, L.; PEI, Y. Cotton flavonoid structural genes related to the pigmentation in brown fibers. Biochemical and Biophysical Research Communications, v. 358, n. 1, p. 7378, 2007.

License information: This is an open-access article distributed under the terms of the Creative Commons Attribution License (type CC-BY), which permits unrestricted use, distribution and reproduction in any medium, provided the original article is properly cited. 